\title{
Fixed-order FIR approximation of linear systems from quantized input and output data
}

\author{
V. Cerone ${ }^{\mathrm{a}}$, D. Piga ${ }^{\mathrm{b}}$, D. Regruto ${ }^{*, a}$ \\ ${ }^{a}$ Dipartimento di Automatica e Informatica, Politecnico di Torino, Corso Duca degli Abruzzi 24, \\ 10129 Torino, Italy \\ ${ }^{b}$ Control Systems Group, Department of Electrical Engineering, Eindhoven University of Technology, \\ Postbus 513, 5600 MB Eindhoven, The Netherlands
}

\begin{abstract}
The problem of identifying a fixed-order FIR approximation of linear systems with unknown structure, assuming that both input and output measurements are subjected to quantization, is dealt with in this paper. A fixed-order FIR model providing the best approximation of the input-output relationship is sought by minimizing the worst-case distance between the output of the true system and the modeled output, for all possible values of the input and output data consistent with their quantized measurements. The considered problem is firstly formulated in terms of robust optimization. Then, two different algorithms to compute the optimum of the formulated problem by means of linear programming techniques are presented. The effectiveness of the proposed approach is illustrated by means of a simulation example.
\end{abstract}

Key words: FIR models, Linear programming, Quantized identification, Robust optimization.

\section{Introduction}

In many engineering applications, only binary-valued or quantized measurement data are available. Typical examples include vision systems which commonly make use of pixelized information; robotics applications where digital rotary or linear encoders are employed to measure position and velocity; wireless sensor networks where signals are

*Corresponding author. email: diego.regruto@polito.it, Phone: +39 0110907077, Fax: +39 0110907099

Email addresses: vito.cerone@polito.it (V. Cerone), D.Piga@tue.nl (D. Piga), diego.regruto@polito.it (D. Regruto)

Preprint submitted to Systems \& Control Letters 
quantized and converted into a finite number of bits before being transmitted through communication channels with limited bandwidth.

Identification of dynamical systems from binary and quantized observations has attracted the attention of many researchers in the last years. More precisely, a maximum likelihood approach for parameter estimation of a static function from binary-valued output data is discussed in [1], while identification of linear dynamical systems which are equipped with only binary-valued sensors is addressed in [2] in the case of stochastic and deterministic description of the disturbances affecting the model. The methodologies discussed in [2] have been extended to system identification with quantized observations [3] and to identification of Wiener models [4]. Identification of linear dynamical systems from quantized output observations is dealt with in $[5,6,7,8,9,10,11]$. In particular, local convergence results in identification of IIR models from binary measurements are given in [10] and extended in [11] for the identification of FIR models from measurements subjected to non-uniform quantization. Global convergence results to a parameter setting corresponding to a perfect input-output model or to the boundary of the chosen model set are also provided. Other approaches for the identification of linear systems from quantized output measurements can be found in [12] and [13], where identification of Wiener-like models with non-invertible nonlinearity is dealt with. The problem of state estimation for linear systems from quantized measurements is considered in [14] and [15]. In the framework of bounded-error identification, that is when disturbances are supposed to be unknown-but-bounded, recent results are presented in [16], [17] and [18] on optimal input design for FIR model identification from binary and quantized observations. The reader is referred to the book [19] and references therein for a detailed discussion on system identification from quantized measurements.

All the papers mentioned above assume that only the output signal is subjected to quantization, while the input of the system is perfectly known. When also the input measurements are quantized, the identification problem can be formulated in terms of errorin-variables problems with unknown-but-bounded measurement noise. In this case the solution to the identification problem can be obtained by applying the results discussed in [20], [21], [22] and [23] where different algorithms are presented to compute bounds on the parameters of IIR models consistent with the assumed model structure, noise bounds and measured data. Linear system identification from quantized input/output (I/O) data 
in the presence of additive measurement noise on the output signal is tackled in [24] and [25] by means of least-square and instrumental-variable approaches. Identification of autoregressive moving average models from binary measurements of the input and output signals is addressed in [26], which provides an algorithm yielding consistent parameter estimates under the assumption that the input/output power ratio of the system is apriori known and white disturbances with known distributions affect the input/output measurements.

The aim of this paper is to evaluate the parameters of a fixed-order FIR model which provides the best worst-case approximation of the (I/O) relationship of a linear system with unknown structure. More precisely, the FIR model parameters are identified in order to minimize the worst-case distance between the output of the true system and the modeled output for all possible values of input and output data consistent with their quantized measurements. The paper is organized as follows. Computation of optimal worst-case FIR parameters is formulated as a robust optimization problem in Section 2. Two different approaches are presented in Section 3 to compute the solution of the formulated robust optimization problem by means of linear programming techniques. Capabilities of the proposed identification scheme, together with a comparison with the standard least square algorithm, are discussed in Section 4.

\section{Problem formulation}

Consider a stable discrete-time single-input single-output linear dynamical system described by

$$
y_{t}=\sum_{t=1}^{\infty} h(t) u_{t},
$$

where $\{h(t)\}_{t=1}^{\infty}$ is the impulse response, while $u_{t}$ and $y_{t}$ are the continuous-amplitude input and output signals at the time instant $t$, respectively. Measurements $u_{t}^{q}$ of the input signal $u_{t}$ are obtained by the following $m_{u}$-level quantizer $\mathcal{Q}_{u}(\cdot)$ :

$$
u_{t}^{q}=\mathcal{Q}_{u}\left(u_{t}\right)= \begin{cases}\left\lfloor\frac{u_{t}-\underline{C}_{u}}{\Delta_{u}}\right\rfloor \Delta_{u}+\underline{C}_{u} & \text { if } \underline{C}_{u}<u_{t}<\bar{C}_{u}, \\ \bar{C}_{u}, & \text { if } u_{t} \geq \bar{C}_{u}, \\ \underline{C}_{u}, & \text { if } u_{t} \leq \underline{C}_{u},\end{cases}
$$

where $\lfloor\cdot\rfloor$ denotes the floor operator, $\left[\underline{C}_{u} ; \bar{C}_{u}\right]$ is the range of the quantizer and $\Delta_{u}=$ $\frac{\bar{C}_{u}-\underline{C}_{u}}{m_{u}-1}$ is the quantization step. Similarly, quantized measurements $y_{t}^{q}$ of the output 
signal $y_{t}$ are given by the quantizer $\mathcal{Q}_{y}(\cdot)$ with $m_{y}$ levels described by

$$
y_{t}^{q}=\mathcal{Q}_{y}\left(y_{t}\right)= \begin{cases}\left\lfloor\frac{y_{t}-\underline{C}_{y}}{\Delta_{y}}\right\rfloor \Delta_{y}+\underline{C}_{y} & \text { if } \underline{C}_{y}<y_{t}<\bar{C}_{y}, \\ \bar{C}_{y}, & \text { if } y_{t} \geq \bar{C}_{y}, \\ \underline{C}_{y}, & \text { if } y_{t} \leq \underline{C}_{y},\end{cases}
$$

with $\Delta_{y}=\frac{\bar{C}_{y}-\underline{C}_{y}}{m_{y}-1}$.

Assumption 1. We assume that quantizers $\mathcal{Q}_{u}(\cdot)$ and $\mathcal{Q}_{y}(\cdot)$ are designed so that the input and the output signals $u_{t}$ and $y_{t}$ belong, respectively, to the quantization ranges $\left[\underline{C}_{u} ; \bar{C}_{u}\right]$ and $\left[\underline{C}_{y} ; \bar{C}_{y}\right]$.

In view of Assumption 1, the unknown noise-free input $u_{t}$ ranges within the open interval $\left[u_{t}\right)$ defined as $\left[u_{t}\right) \doteq\left[u_{t}^{q} ; u_{t}^{q}+\Delta_{u}\right)$, while the unknown noise-free output $y_{t}$ takes values in the open set $\left[y_{t}\right) \doteq\left[y_{t}^{q} ; y_{t}^{q}+\Delta_{y}\right)$.

For a given integer $n \geq 1$ and for a given sequence of input and output quantized measurements $\mathbf{u}^{q}=\left[u_{-n}^{q}, u_{-n+1}^{q}, \ldots, u_{N}^{q}\right]^{\mathrm{T}}$ and $\mathbf{y}^{q}=\left[y_{1}^{q}, y_{2}^{q}, \ldots, y_{N}^{q}\right]^{\mathrm{T}}$, the aim of our work is to estimate the parameters $\theta=\left[\theta_{0}, \theta_{1}, \ldots, \theta_{n}\right]^{\mathrm{T}}$ of an $n$-order finite-impulseresponse (FIR) model $\mathcal{F}$ :

$$
\mathcal{F}: \hat{y}_{t}=\sum_{k=0}^{n} \theta_{k} u_{t-k} .
$$

Remark 1. Note that, since a finite record of input/output data is collected, Assumption 1 can be relaxed by assuming that $u_{t} \in\left[\underline{C}_{u} ; \bar{C}_{u}\right]$ only for the time indexes $t=-n,-n+$ $1, \ldots, N$ and $y_{t} \in\left[\underline{C}_{y} ; \bar{C}_{y}\right]$ for $t=1, \ldots, N$. Indeed, rough a-priori information on the upper/lower bound of the input signal can be used to calibrate the quantizer $\mathcal{Q}_{u}(\cdot)$ in order to satisfy Assumption 1. Nevertheless, if such rough a-priori information is not available, the user can calibrate the quantizer $\mathcal{Q}_{u}(\cdot)$ by gradually increasing the quantization range $\left[\underline{C}_{u} ; \bar{C}_{u}\right]$, until there is no value of the measured inputs $u_{t}^{q}$ equal to $\underline{C}_{u}$ or $\bar{C}_{u}$. If fact, if there is no value of $u_{t}^{q}$ equal to $\underline{C}_{u}$ or $\bar{C}_{u}$, then the true input signal $u_{t}$ is guaranteed to belong to the interval $\left[\underline{C}_{u} ; \bar{C}_{u}\right]$. The same considerations can be applied in order to calibrate the output quantizer $\mathcal{Q}_{y}(\cdot)$.

The FIR model $\mathcal{F}$ is computed in order to minimize the worst-case distance between the noise-free output sequence $\mathbf{y}=\left[y_{1}, y_{2}, \ldots, y_{N}\right]^{\mathrm{T}}$ and the predicted output $\hat{\mathbf{y}}=\left[\hat{y}_{1}, \hat{y}_{2}, \ldots, \hat{y}_{N}\right]^{\mathrm{T}}$ for all possible admissible values of the noise-free input 
$\mathbf{u}=\left[u_{-n}, u_{-n+1}, \ldots, u_{N}\right]^{\mathrm{T}}$ and output signals $\mathbf{y}$, that is, for all values of $u_{k} \in\left[u_{k}\right)$ and $y_{t} \in\left[y_{t}\right)$, with $k=-n, \ldots, N$ and $t=1, \ldots, N$. Thus, the optimal worst-case parameters $\theta^{*}$ can be computed by solving the following optimization problem:

$$
\begin{gathered}
\theta^{*}=\arg \min _{\theta} \quad \max _{u_{k} \in\left[u_{k}\right]} \quad\|\hat{\mathbf{y}}-\mathbf{y}\|_{\infty} \\
y_{t} \in\left[y_{t}\right] \\
k=-n, \ldots, N \\
t=1, \ldots, N
\end{gathered}
$$

where $\left[u_{k}\right]$ and $\left[y_{k}\right]$ denote the closure of the open set $\left[u_{k}\right)$ and $\left[y_{k}\right)$, respectively, that is $\left[u_{t}\right] \doteq\left[u_{t}^{q} ; u_{t}^{q}+\Delta_{u}\right]$ and $\left[y_{t}\right] \doteq\left[y_{t}^{q} ; y_{t}^{q}+\Delta_{y}\right]$, while $\|\hat{\mathbf{y}}-\mathbf{y}\|_{\infty}$ is the $\infty$-norm of vector $\hat{\mathbf{y}}-\mathbf{y}$

Remark 2. Problem (5) can be interpreted as a game between designer and nature, where the designer has to look for a strategy (FIR parameters $\theta$ ) which minimizes a given criterion $\left(\|\hat{\boldsymbol{y}}-\boldsymbol{y}\|_{\infty}\right)$ against the nature, which in turn plays the most disadvantageous strategy for the designer (looking for the values of $u_{t} \in\left[u_{t}\right]$ and $y_{t} \in\left[y_{t}\right]$ maximizing $\left.\|\hat{\boldsymbol{y}}-\boldsymbol{y}\|_{\infty}\right)$. The interested reader is referred to [27] for an overview of the main principles of game theory.

Remark 3. If the 2-norm is considered in the objective function of Problem (5), the deterministic total least square (TLS) approach described in [28] (Chapter 2.8) could be used to solve the minmax problem (5). However, although by means of the TLS approach it is possible to deal with the case of bounded input and output errors, the solution computed by applying the deterministic TLS method described in [28] is, in general, affected by some conservativeness. In fact, the algorithm proposed in [28] is based on the implicit assumption that the uncertain variables affecting the different rows of the regressor are independent with each others. Unfortunately, that is not the case in Problem (5) where the uncertainties affecting the rows of the regressors are correlated, due to the presence of different occurrences of the same uncertain input samples. On the contrary, in this paper we propose two different methods to exactly solve Problem (5) by taking into account such a correlation. 


\section{Computation of optimal worst-case FIR parameters $\theta^{*}$}

In this section we present two different approaches to compute the optimal worst-case parameters $\theta^{*}$ by means of linear programming optimization.

First, we note that, by introducing the slack variable $\xi$ and by substituting equation (4) into (5), problem (5) can be written as the following robust optimization problem

$$
\begin{aligned}
& \left(\theta^{*}, \xi^{*}\right)=\arg \min _{\theta, \xi} \xi \\
& \text { s.t. } \\
& -\xi \leq \sum_{k=0}^{n} \theta_{k} u_{t-k}-y_{t} \leq \xi, \forall u_{k} \in\left[u_{k}\right], \forall y_{t} \in\left[y_{t}\right] \\
& k=-n, \ldots, N ; \quad t=1, \ldots, N .
\end{aligned}
$$

Note that in (6) there are infinitely many constraints, since the inequalities $-\xi \leq$ $\sum_{k=0}^{n} \theta_{k} u_{t-k}-y_{t} \leq \xi$ must be satisfied for all possible values of $u_{k}$ and $y_{t}$ in the uncertainty intervals $\left[u_{k}\right]$ and $\left[y_{t}\right]$, respectively. This kind of problems are known as robust optimization problems or semi-infinite programming problems, namely problems with finitely many decision variables and infinitely many constraints [29]. In the following, two different techniques are presented to reduce (6) to a linear programming problem with a finite number of constraints. These two methods will be referred to as vertex approach and nonnegative-scalar approach.

\subsection{Vertex-approach}

Let $\mathcal{B}_{t}$ be an $n+2$-dimensional box defined as

$$
\mathcal{B}_{t}=\left[u_{t}\right] \times\left[u_{t-1}\right] \times \ldots \times\left[u_{t-n}\right] \times\left[y_{t}\right]
$$

and $b_{t}=\left[u_{t}, u_{t-1}, \ldots, u_{t-n}, y_{t}\right]$ be an element of $\mathcal{B}_{t}$. Let $b_{t}^{j}$, with $j=1, \ldots, 2^{n+2}$, be the $j$-th vertex of $\mathcal{B}_{t}$.

Proposition 1. Condition

$$
-\xi \leq \sum_{k=0}^{n} \theta_{k} u_{t-k}-y_{t} \leq \xi
$$

holds for all $u_{t-k} \in\left[u_{t-k}\right]$ and for all $y_{t} \in\left[y_{t}\right]$, with $k=0, \ldots, n$ if and only if

$$
-\xi \leq \sum_{k=0}^{n} \theta_{k} b_{t, k+1}^{j}-b_{t, n+2}^{j} \leq \xi, \quad \forall j=1, \ldots, 2^{n+2}
$$


where $b_{t, k}^{j}$ denotes the $k$-th component of the vertex $b_{t}^{j}$.

Proof The proof of Proposition 1 follows from the fact that maximum/minimum of $\sum_{k=0}^{n} \theta_{k} u_{t-k}-y_{t}$ is attained at some vertex $b_{t}^{j}$ of box $\mathcal{B}_{t}$. In particular, for a given $\theta$, the maximum of $\sum_{k=0}^{n} \theta_{k} u_{t-k}-y_{t}$ over $\mathcal{B}_{t}$ is

$$
\sum_{k=0}^{n} \theta_{k}\left(u_{t-k}^{q}+I_{\theta_{k}} \Delta_{u}\right)-y_{t}
$$

while the minimum value is

$$
\sum_{k=0}^{n} \theta_{k}\left[u_{t-k}^{q}+\left(1-I_{\theta_{k}}\right) \Delta_{u}\right]-\left(y_{t}+\Delta_{y}\right)
$$

where $I_{\theta_{k}}$ is defined as

$$
I_{\theta_{k}}= \begin{cases}1 & \text { if } \theta_{k} \geq 0 \\ 0 & \text { if } \theta_{k}<0\end{cases}
$$

On the basis of Proposition 1, the optimal worst-case FIR parameters $\theta^{*}$ solution to problem (6), can be obtained by solving the following linear programming problem:

$$
\begin{aligned}
& \left(\theta^{*}, \xi^{*}\right)=\arg \min _{\theta, \xi} \xi \\
& \text { s.t. } \\
& -\xi \leq \sum_{k=0}^{n} \theta_{k} b_{t, k+1}^{j}-b_{t, n+2}^{j} \leq \xi, \forall j=1, \ldots, 2^{n+2}, \quad t=1, \ldots, N .
\end{aligned}
$$

It is worth remarking that the number $M$ of linear constraints involved in (11) is $M=2 N 2^{n+2}$. Thus, since $M$ increases exponentially with the order $n$ of the FIR model $\mathcal{F}$, application of the presented procedure is limited to small values of $n$. In order to compute a solution of problem (6) also for large values of $n$, in the following we present an alternative method which leads to a linear programming problem whose number of constraints increases linearly with $n$.

\subsection{Nonnegative-scalar approach}

Let us rewrite the following robust constraint appearing in the optimization problem (6)

$$
-\xi \leq \sum_{k=0}^{n} \theta_{k} u_{t-k}-y_{t} \leq \xi, \quad \forall y_{t} \in\left[y_{t}\right] ; \quad \forall u_{t-k} \in\left[u_{t-k}\right], \quad k=0, \ldots, n,
$$


as

$$
\left\{\begin{array}{l}
\sum_{k=0}^{n} \theta_{k} u_{t-k}-y_{t}^{q} \leq \xi \\
\sum_{k=0}^{n} \theta_{k} u_{t-k}-\left(y_{t}^{q}+\Delta_{y}\right) \geq-\xi \\
\forall u_{t-k} \in\left[u_{t-k}\right], \quad k=0, \ldots, n
\end{array}\right.
$$

or equivalently in terms of nonnegative robust constraints, i.e.

$$
\left\{\begin{array}{l}
-\sum_{k=0}^{n} \theta_{k} u_{t-k}+y_{t}^{q}+\xi \geq 0 \\
\sum_{k=0}^{n} \theta_{k} u_{t-k}-y_{t}^{q}-\Delta_{y}+\xi \geq 0 \\
\forall u_{t-k} \in\left[u_{t-k}\right], \quad k=0, \ldots, n
\end{array}\right.
$$

It is worth remarking that only $\theta_{k}$ and $\xi$ are the decision variables in (14), while $u_{t-k}$ is an uncertain variable which is assumed to belong to the uncertainty set $\left[u_{t-k}\right]$. This means that $\theta_{k}$ and $\xi$ have to be computed so that the constraints $-\sum_{k=0}^{n} \theta_{k} u_{t-k}+y_{t}^{q}+\xi \geq$ 0 and $\sum_{k=0}^{n} \theta_{k} u_{t-k}-y_{t}^{q}-\Delta_{y}+\xi \geq 0$ are satisfied for all possible values of $u_{t-k}$ in $\left[u_{t-k}\right]$.

Proposition 2. The collection of robust constraints

$$
\left\{\begin{array}{l}
-\sum_{k=0}^{n} \theta_{k} u_{t-k}+y_{t}^{q}+\xi \geq 0, \forall u_{t-k} \in\left[u_{t-k}\right], \\
k=0, \ldots, n ; \quad t=1, \ldots, N
\end{array}\right.
$$

is equivalent to the following set of constraints:

$$
\left\{\begin{array}{l}
-\sum_{k=0}^{n} \theta_{k} u_{t-k}+y_{t}^{q}+\xi= \\
=\underline{\rho}_{t}+\sum_{k=0}^{n} \underline{\lambda}_{k}\left(-u_{t-k}^{q}+u_{t-k}\right)+\sum_{k=0}^{n} \underline{\sigma}_{k}\left(u_{t-k}^{q}+\Delta_{u}-u_{t-k}\right) \\
\text { for some } \underline{\lambda}_{k} \geq 0 ; \underline{\sigma}_{k} \geq 0 ; \underline{\rho}_{t} \geq 0 \\
k=0, \ldots, n ; \quad t=1, \ldots, N
\end{array}\right.
$$

Proof We first prove that (16) implies (15). Indeed, for all $u_{t-k} \in\left[u_{t-k}\right],-u_{t-k}^{q}+u_{t-k} \geq$ 0 and $u_{t-k}^{q}+\Delta_{u}-u_{t-k} \geq 0$. Besides, since $\underline{\lambda}_{k} \geq 0, \underline{\sigma}_{k} \geq 0$ and $\underline{\rho}_{t} \geq 0$ for all $k=0, \ldots, n$ and for all $t=1, \ldots, N$, the right side of the equality in (16) is always positive, therefore

$$
-\sum_{k=0}^{n} \theta_{k} u_{t-k}+y_{t}^{q}+\xi \geq 0
$$

for any $u_{t-k} \in\left[u_{t-k}\right]$, with $k=0 ; \ldots, n$. Thus, condition (15) holds.

In order to prove that (15) implies (16) we have to show that, when (15) holds, there exists some nonnegative constants $\underline{\rho}_{t} \underline{\lambda}_{k}, \underline{\sigma}_{k}$, with $k=0, \ldots, n$ and $t=1, \ldots, N$ such that the terms $-\sum_{k=0}^{n} \theta_{k} u_{t-k}+y_{t}^{q}+\xi$, with $t=1, \ldots, N$, can be written as

$$
\underline{\rho}_{t}+\sum_{k=0}^{n} \underline{\lambda}_{k}\left(-u_{t-k}^{q}+u_{t-k}\right)+\sum_{k=0}^{n} \underline{\sigma}_{k}\left(u_{t-k}^{q}+\Delta_{u}-u_{t-k}\right) .
$$


Such constants are equal to

$$
\begin{gathered}
\underline{\lambda}_{k}= \begin{cases}0, & \text { if } \theta_{k}>0 \\
-\theta_{k}, & \text { if } \theta_{k} \leq 0\end{cases} \\
\underline{\sigma}_{k}= \begin{cases}\theta_{k}, & \text { if } \theta_{k}>0 \\
0, & \text { if } \theta_{k} \leq 0\end{cases} \\
\underline{\rho}_{t}=-\sum_{k=0}^{n} \theta_{k}\left(u_{t-k}^{q}+\mathcal{I}_{\theta_{k}} \Delta_{u}\right)+y_{t}^{q}+\xi,
\end{gathered}
$$

with $\mathcal{I}_{\theta_{k}}$ defined in (10). Indeed, $\underline{\lambda}_{k}$ and $\underline{\sigma}_{k}$ in (19) and (20) are nonnegative by definition. Besides, from eq. (15), the term $-\sum_{k=0}^{n} \theta_{k} u_{t-k}+y_{t}^{q}+\xi$ is nonnegative for all $u_{t-k} \in\left[u_{t-k}\right]$, thus also $\underline{\rho}_{t}$ is nonnegative since $u_{t-k}^{q}+\mathcal{I}_{\theta_{k}} \Delta_{u} \in\left[u_{t-k}\right]$. By substituting $\underline{\lambda}_{k}, \underline{\sigma}_{k}$ and $\underline{\rho}_{t}$ defined in (19)-(21) into eq. (18), the term in (18) becomes $-\sum_{k=0}^{n} \theta_{k} u_{t-k}+y_{t}^{q}+\xi$. This completes the proof.

Remark 4. The equality constraint appearing in (16) is an equality between two polynomials in the variables $u_{t-k}$ (with $k=0, \ldots, n$ ), namely $\sum_{k=0}^{n} \theta_{k} u_{t-k}-y_{t}^{q}-\Delta_{y}+\xi$, and $\bar{\rho}_{t}+\sum_{k=0}^{n} \bar{\lambda}_{k}\left(u_{t-k}-u_{t-k}^{q}\right)+\sum_{k=0}^{n} \bar{\sigma}_{k}\left(u_{t-k}^{q}+\Delta_{u}-u_{t-k}\right)$. As is well known, two polynomials are equivalent if and only if the coefficients of the corresponding powers are equal. Furthermore, the coefficients of the polynomials in (16) depend on the unknowns $\theta, \xi$, $\bar{\lambda}_{k}, \bar{\sigma}_{k}$ and $\bar{\rho}_{t}$ (with $k=0, \ldots, n$ and $\left.t=1, \ldots, N\right)$. Therefore, the equivalence of the polynomials in (16) leads to a set of equality constraints in the variables $\theta, \xi, \bar{\lambda}_{k}$, $\bar{\sigma}_{k}$ and $\bar{\rho}_{t}$.

Proposition 3. The set of robust constraints

$$
\left\{\begin{array}{l}
\sum_{k=0}^{n} \theta_{k} u_{t-k}-y_{t}^{q}-\Delta_{y}+\xi \geq 0, \forall u_{t-k} \in\left[u_{t-k}\right] \\
k=0, \ldots, n ; t=1, \ldots, N
\end{array}\right.
$$


is equivalent to the following set of constraints:

$$
\left\{\begin{array}{l}
\sum_{k=0}^{n} \theta_{k} u_{t-k}-y_{t}^{q}-\Delta_{y}+\xi= \\
=\bar{\rho}_{t}+\sum_{k=0}^{n} \bar{\lambda}_{k}\left(-u_{t-k}^{q}+u_{t-k}\right)+\sum_{k=0}^{n} \bar{\sigma}_{k}\left(u_{t-k}^{q}+\Delta_{u}-u_{t-k}\right) \\
\text { for some } \bar{\lambda}_{k} \geq 0 ; \bar{\sigma}_{k} \geq 0 ; \bar{\rho}_{t} \geq 0 \\
k=0, \ldots, n ; \quad t=1, \ldots, N .
\end{array}\right.
$$

Proof Proof of Proposition 3 follows by considerations similar to the ones used in the proof of Proposition 2. In this case, the values of $\bar{\lambda}_{k}, \bar{\sigma}_{k}$ and $\bar{\rho}_{t}$ which satisfy conditions in (23) are given by

$$
\begin{gathered}
\bar{\lambda}_{k}= \begin{cases}\theta_{k}, & \text { if } \theta_{k}>0, \\
0, & \text { if } \theta_{k} \leq 0,\end{cases} \\
\bar{\sigma}_{k}= \begin{cases}0, & \text { if } \theta_{k}>0, \\
-\theta_{k}, & \text { if } \theta_{k} \leq 0,\end{cases} \\
\bar{\rho}_{t}=\sum_{k=0}^{n} \theta_{k}\left(u_{t-k}^{q}+\left(1-\mathcal{I}_{\theta_{k}}\right) \Delta_{u}\right)-y_{t}^{q}-\Delta_{y}+\xi .
\end{gathered}
$$

On the basis of Propositions 2 and 3, the optimal worst-case FIR parameters $\theta^{*}$ solution to problem (6), can be obtained by solving the following optimization problem:

$$
\begin{array}{rc}
\left(\theta^{*}, \xi^{*}\right)=\arg & \min \\
& \theta, \xi \\
& \bar{\lambda}_{k}, \bar{\sigma}_{k}, \bar{\rho}_{t} \\
& \underline{\lambda}_{k}, \underline{\sigma}_{k}, \underline{\rho}_{t}
\end{array}
$$

s.t.

$$
\begin{aligned}
& \sum_{k=0}^{n} \theta_{k} u_{t-k}-y_{t}^{q}-\Delta_{y}+\xi=\bar{\rho}_{t}+\sum_{k=0}^{n} \bar{\lambda}_{k}\left(u_{t-k}-u_{t-k}^{q}\right)+\sum_{k=0}^{n} \bar{\sigma}_{k}\left(u_{t-k}^{q}+\Delta_{u}-u_{t-k}\right) \\
& -\sum_{k=0}^{n} \theta_{k} u_{t-k}+y_{t}^{q}+\xi=\underline{\rho}_{t}+\sum_{k=0}^{n} \underline{\lambda}_{k}\left(-u_{t-k}^{q}+u_{t-k}\right)+\sum_{k=0}^{n} \underline{\sigma}_{k}\left(u_{t-k}^{q}+\Delta_{u}-u_{t-k}\right)
\end{aligned}
$$

for some $\bar{\lambda}_{k} \geq 0 ; \bar{\sigma}_{k} \geq 0 ; \bar{\rho}_{t} \geq 0$;

$$
\underline{\lambda}_{k} \geq 0 ; \underline{\sigma}_{k} \geq 0 ; \underline{\rho}_{t} \geq 0 ;
$$$$
k=0, \ldots, n ; \quad t=1, \ldots, N \text {. }
$$

Property 1. Optimization problem (27) is a linear programming problem in the decision variables $\theta, \xi, \bar{\lambda}_{k}, \bar{\sigma}_{k}, \bar{\rho}_{t}, \underline{\lambda}_{k}, \underline{\sigma}_{k}, \underline{\rho}_{t}$. 
Proof As highlighted in Remark 4, the polynomial $\sum_{k=0}^{n} \theta_{k} u_{t-k}-y_{t}^{q}-\Delta_{y}+\xi$ (in the variables $u_{t-k}$ (with $\left.k=0, \ldots, n\right)$ ) is equivalent to the polynomial

$$
\bar{\rho}_{t}+\sum_{k=0}^{n} \bar{\lambda}_{k}\left(u_{t-k}-u_{t-k}^{q}\right)+\sum_{k=0}^{n} \bar{\sigma}_{k}\left(u_{t-k}^{q}+\Delta_{u}-u_{t-k}\right)
$$

if and only if the coefficients of the corresponding powers are equal. This leads to linear constraints in the variables $\theta, \xi, \bar{\lambda}_{k}, \bar{\sigma}_{k}$ and $\bar{\rho}_{t}$, with $k=0, \ldots, n$ and $t=1, \ldots, N$. The same considerations hold in order to impose that the polynomial $-\sum_{k=0}^{n} \theta_{k} u_{t-k}+y_{t}^{q}+\xi$ is equivalent to polynomial

$$
\underline{\rho}_{t}+\sum_{k=0}^{n} \underline{\lambda}_{k}\left(-u_{t-k}^{q}+u_{t-k}\right)+\sum_{k=0}^{n} \underline{\sigma}_{k}\left(u_{t-k}^{q}+\Delta_{u}-u_{t-k}\right) .
$$

Remark 5. The feasible set of problem (27) is defined by $M=4 N+2 n$ linear constraints, thus $M$ increases linearly with the order $n$ of the FIR $\mathcal{F}$. Therefore, unlike the "vertex" approach, the "nonnegative-scalar" approach can be efficiently applied also in the case of FIR models $\mathcal{F}$ with large order $n$.

Remark 6. It is worth remarking that both the vertex and the nonnegative-scalar approach can be applied, with minor modifications, also in the case the input and the output signals are measured by means of centered quantizers described by:

$$
\begin{gathered}
u_{t}^{q}=\mathcal{Q}_{u}\left(u_{t}\right)= \begin{cases}\operatorname{round}\left(\frac{u_{t}-\underline{C}_{u}}{\Delta_{u}}\right) \Delta_{u}+\underline{C}_{u} & \text { if } \underline{C}_{u}<u_{t}<\bar{C}_{u}, \\
\bar{C}_{u}, & \text { if } u_{t} \geq \bar{C}_{u}, \\
\underline{C}_{u}, & \text { if } u_{t} \leq \underline{C}_{u},\end{cases} \\
y_{t}^{q}=\mathcal{Q}_{y}\left(y_{t}\right)= \begin{cases}\operatorname{round}\left(\frac{y_{t}-\underline{C}_{y}}{\Delta_{y}}\right) \Delta_{y}+\underline{C}_{y} & \text { if } \underline{C}_{y}<y_{t}<\bar{C}_{y}, \\
\bar{C}_{y}, & \text { if } y_{t} \geq \bar{C}_{y}, \\
\underline{C}_{y}, & \text { if } y_{t} \leq \underline{C}_{y},\end{cases}
\end{gathered}
$$

where round $(\cdot)$ denotes the closest integer approximation. Extension of the proposed approaches to the case of nonuniform quantization is also straightforward. 


\section{Simulation example}

In this section we show the performance of the discussed approach through a numerical example. The true system generating the data is an IIR model described by

$$
y_{t}=a_{1} y_{t-1}+a_{2} y_{t-2}+b_{0} u_{t}+b_{1} u_{t-1}+b_{2} u_{t-2}
$$

with $a_{1}=0.15, a_{2}=0.60, b_{0}=-0.10 b_{1}=3.50, b_{2}=-2.10$. The input signal $u_{t}$ is a random variable uniformly distributed in $[-0.2 ; 0.2]$. Quantized measurements of the input and of the output signal are obtained by means of uniform and centered quantizers described by (28)-(29) with range $\left[\underline{C}_{u} ; \bar{C}_{u}\right]=\left[\begin{array}{ll}-0.2 ; & 0.2\end{array}\right]$ and $\left[\underline{C}_{y} ; \bar{C}_{y}\right]=[-2 ; 2]$, respectively. In order to show the effect of the input quantization error, four different input quantizers with levels $m_{u}=4,6,8,10$ are considered. The signal-to-noise ratios $S N R_{u}=10 \log _{10}\left(\frac{\sum_{t=1}^{N}\left(u_{t}\right)^{2}}{\sum_{t=1}^{N}\left(u_{t}-u_{t}^{q}\right)^{2}}\right)$, corresponding to the considered quantization levels $m_{u}$, are reported in Table 1. Similarly, four different output quantizers with levels $m_{y}=8,12,16,20$ are considered in order to analyze the effect of the output quantization error. Table 2 shows the values of the signal-to-noise ratios $S N R_{y}=10 \log _{10}\left(\frac{\sum_{t=1}^{N}\left(y_{t}\right)^{2}}{\sum_{t=1}^{N}\left(y_{t}-y_{t}^{q}\right)^{2}}\right)$ corresponding to the considered output quantization levels $m_{y}$. It worth pointing out that, in order to provide representative results, the input and the output quantizers are chosen to be different with each other. In fact, for input quantization levels $m_{u}=8,12,16,20$, the corresponding signal-to-noise rations are higher than $20 \mathrm{~dB}$ and the obtained results would not clearly show the effect of the noise on the input measurements. All possible combinations of the input and the output quantizers are analyzed in the simulation example. Furthermore, for each combination of the input and the output quantizers, a MonteCarlo simulation with 100 runs is performed with a new input realization in each run. The optimal worst-case parameters $\theta^{*}$ of a FIR model $\mathcal{F}^{*}$ of order $n=30$ are estimated, at each MonteCarlo run, from an input/ouput sequence of length $N=200$. It is worth remarking that, in the considered

Table 1: Signal-to-noise ratio $\left(S N R_{u}\right)$ on the input signal measurements vs number of quantization levels $m_{u}$.

\begin{tabular}{|c||cccc|}
\hline$m_{u}$ & 4 & 6 & 8 & 10 \\
\hline$S N R_{u}$ & $4 \mathrm{~dB}$ & $11 \mathrm{~dB}$ & $21 \mathrm{~dB}$ & $32 \mathrm{~dB}$ \\
\hline
\end{tabular}


example, the feasible set of optimization problem (11) is described by $2 N 2^{n+2} \simeq 1.7 \cdot 10^{12}$ constraints. Therefore, because of high computational complexity, the vertex approach discussed in Section 3.1 can not be exploited to compute FIR parameters $\theta^{*}$. On the other hand, the use of the nonnegative-scalar method leads to optimization problem (27) with $4 N+2 n=860$ constraints, that can be efficiently solved by means of linear programming solvers like Matlab function linprog. The performance of the estimated FIR model is tested on a validation set with $N_{v a l}=50$ input/output measurements. The validation mean square error $M S E_{V}$, defined as

$$
M S E_{V}=\frac{1}{N_{v a l}} \sum_{t=1}^{N_{v a l}}\left(\hat{y}_{t}-y_{t}\right)^{2},
$$

is used to evaluate the matching between real data $y_{t}$ and estimated data $\hat{y}_{t}$. Besides, performances of the identified FIR model $\mathcal{F}^{*}$ with parameters $\theta^{*}$ are also compared with performances of a FIR model $\mathcal{F}_{L S}$ of the same order $n$, whose parameters $\theta_{L S}$ are computed by means of standard least-square (LS) estimation, that is

$$
\theta_{L S}=\arg \min _{\theta} \sum_{t=1}^{N}\left(\sum_{k=0}^{n} \theta_{k} u_{t-k}^{q}-y_{t}^{q}\right)^{2} .
$$

Fig. 1 shows the real output signal $y_{t}$ in the validation data set and the estimated output $\hat{y}_{t}$ of FIR models $\mathcal{F}^{*}$ and $\mathcal{F}_{L S}$ obtained in one of the Monte Carlo simulation runs with quantization levels $m_{u}=4$ and $m_{y}=12$. The magnitude of the errors between real output signal $y_{t}$ and outputs $\hat{y}_{t}$ of FIR models $\mathcal{F}^{*}$ and $\mathcal{F}_{L S}$ is shown in Fig. 2. Figs. 1 and 2 show that the optimal worst-case FIR model $\mathcal{F}^{*}$ achieves better performance with respect to the performance of model $\mathcal{F}_{L S}$. In fact, the $M S E_{V}$ obtained by using FIR model $\mathcal{F}^{*}$ is 0.0109 , smaller than the $M S E_{V}$ obtained by the FIR model $\mathcal{F}_{L S}$, which is equal to 0.0478 . The mean value of the validation mean square error $M S E_{V}$ obtained over the 100 Monte Carlo runs are reported in Table 3. Such results show that, for low values of the signal-to-noise ratios on the input measurements (i.e., $m_{u}=4,6$ ) optimal worst-case FIR model $\mathcal{F}^{*}$ achieves better performance, in terms of $M S E_{V}$, with respect

Table 2: Signal-to-noise ratio $\left(S N R_{y}\right)$ on the output signal measurements vs number of quantization levels $m_{y}$.

\begin{tabular}{|c||cccc|}
\hline$m_{y}$ & 8 & 12 & 16 & 20 \\
\hline$S N R_{y}$ & $6 \mathrm{~dB}$ & $10 \mathrm{~dB}$ & $13 \mathrm{~dB}$ & $16 \mathrm{~dB}$ \\
\hline
\end{tabular}


to the performance of model $\mathcal{F}_{L S}$. The reason is due to the fact that the least-square approach provides a poor estimate in case of noise-corrupted measurements of the input signal. On the other hand, for high values of the signal-to-noise ratios on the input measurements (i.e., $m_{u}=8,10$ ) the performance of the two estimated models are quite similar. It is worth remarking that when non-centered quantizers are used to measure the output signal, the performance of the LS approach significantly drop because of the nonzero average on the measurement noise. On the other hand, the optimal worst-case approach presented in the paper is not affected by this drawback since no assumption on the mean value of the quantization error is made.

\section{Conclusions}

The paper deals with the approximation problem of linear dynamical systems with a fixed-order FIR model from input/output measurements subjected to quantization. Parameters of a FIR model which minimize the worst-case distance between the true output signal and the estimated output, for all possible values of the input signal, are

Table 3: Mean value $\overline{M S E_{V}}$ of the validation mean square error $M S E_{V}$ obtained over the 100 Monte Carlo runs with the FIR models $\mathcal{F}^{*}$ and $\mathcal{F}_{L S}$ and for all possible combinations of the quantizers used to measure the input/output signals.

\begin{tabular}{cc|cc}
\hline$m_{u}$ & $m_{y}$ & $\overline{M S E_{V}}\left(\mathcal{F}^{*}\right)$ & $\overline{M S E_{V}}\left(\mathcal{F}_{L S}\right)$ \\
\hline 4 & 8 & 0.0276 & 0.0522 \\
4 & 12 & 0.0185 & 0.0508 \\
4 & 16 & 0.0113 & 0.0488 \\
4 & 20 & 0.0104 & 0.0475 \\
6 & 8 & 0.0097 & 0.0196 \\
6 & 12 & 0.0092 & 0.0152 \\
6 & 16 & 0.0084 & 0.0138 \\
6 & 20 & 0.0082 & 0.0117 \\
8 & 8 & 0.0078 & 0.0075 \\
8 & 12 & 0.0069 & 0.0067 \\
8 & 16 & 0.0057 & 0.0056 \\
8 & 20 & 0.0055 & 0.0052 \\
10 & 8 & 0.0075 & 0.0076 \\
10 & 12 & 0.0048 & 0.0045 \\
10 & 16 & 0.0043 & 0.0040 \\
10 & 20 & 0.0039 & 0.0036 \\
\hline
\end{tabular}




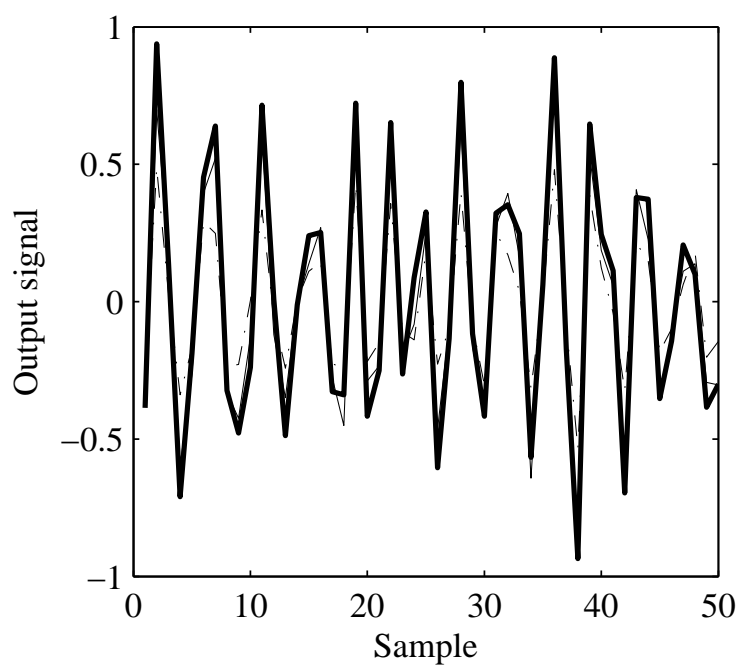

Figure 1: Comparison between real output data (solid thick line), estimated output of filter $\mathcal{F}^{*}$ (solid thin line), estimated output of filter $\mathcal{F}_{L S}$ (dashed line).

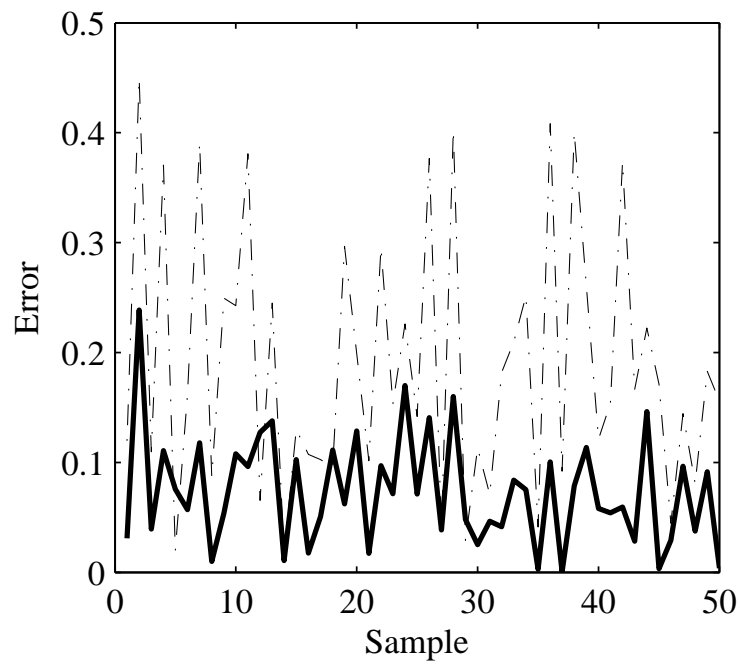

Figure 2: Absolute value of the error between real data and estimated data of filter $\mathcal{F}^{*}$ (solid thick line) and error between real data and estimated data of filter $\mathcal{F}_{L S}$ (dashed line).

computed. The considered approximation problem is formulated in terms of robust optimization and two different methods are discussed in order to compute the solution of the formulated robust optimization problem. The first method, referred to as vertex 
approach, requires the solution to a linear programming problem with a number of constraints which exponentially increases with the order of the FIR model. Thus, because of high computational burden, the vertex approach can be exploited only for small values of the FIR order. The second method, referred to as nonnegative-scalar approach, leads to a linear programming problem with a number of constraints which linearly increases with the order of the FIR model to be estimated. Therefore, the nonnegative-scalar approach can be efficiently used to identify FIR models with high order. The reported simulation example shows that the proposed identification algorithm provides a satisfactory FIR approximation of the IIR data-generating system. In addition, the ability of the proposed worst-case approach to deal with the presence of quantization error is highlighted by comparing the response of the obtained FIR model with the response of the FIR model estimated by means of the standard least squares approach.

\section{Acknowledgments}

This research was developed while Dr. D. Piga was a Ph.D student at the Politecnico di Torino.

\section{References}

[1] E. Rafajlowicz, System identification from cheap, qualitative output observations, IEEE Trans. Automatic Control 41 (1996) 1381-1385.

[2] L. Wang, J. F. Zhang, G. G. Yin, System identification using binary sensors, IEEE Trans. Automatic Control 48 (2003) 1892-1907.

[3] L. Y. Wang, G. G. Yin, Asymptotically efficient parameter estimation using quantized output observations, Automatica 43 (2007) 1178-1191.

[4] Y. Zhao, L. Y. Wang, G. G. Yin, J. F. Zhang, Identification of wiener systems with binary-valued output observations, Automatica 43 (2007) 1752-1765.

[5] E. Weyer, S. Ko, M. C. Campi, Finite sample properties of system identification with quantized output data, in: 48th IEEE Conference on Decision and Control, Shanghai, China, pp. 1532-1537.

[6] E. Colinet, J. Juillard, A weighted least-squares approach to parameter estimation problems based on binary measurements, IEEE Trans. Automatic Control 55 (2010) 148-152.

[7] B. Godoy, G. Goodwin, J. Agüero, D. Marelli, T. Wigren, On identification of FIR systems having quantized output data, Automatica (2011) 1905-1915.

[8] D. Marelli, K. You, M. Fu, Identification of arma models using intermittent and quantized output observations, in: 2011 IEEE International Conference on Acoustics, Speech and Signal Processing (ICASSP), IEEE, pp. 4076-4079.

[9] X. Liu, J. Wang, Q. Zhang, A quadratic-programming-based method to quantized system identification, in: Proc. of IFAC world congress, Milan, Italy, pp. 9052-9057. 
[10] T. Wigren, Approximate gradients, convergence and positive realness in recursive identification of a class of non-linear systems, International Journal of Adaptive Control and Signal Processing 9 (1995) 325-354.

[11] T. Wigren, Adaptive filtering using quantized output measurements, IEEE Transactions on Signal Processing 46 (1998) 3423-3426.

[12] E. W. Bai, J. Reyland, Towards identification of Wiener systems with the least amount of a priori information on the nonlinearity, Automatica 44 (2008) 910-919.

[13] E. W. Bai, J. Reyland, Towards identification of Wiener systems with the least amount of a priori information: IIR cases, Automatica 45 (2009) 956-964.

[14] E. Sviestins, T. Wigren, Optimal recursive state estimation with quantized measurements, IEEE Trans. Automatic Control 45 (2000) 762-767.

[15] Z. Duan, V. P. Jilkov, X. R. Li, State estimation with quantized measurements: Approximate mmse approach, in: 11th International Conference on Information Fusion, pp. 1-6.

[16] M. Casini, A. Garulli, A. Vicino, Optimal input design for identification of systems with quantized measurements, in: 47th IEEE Conference on Decision and Control, pp. 5506-5512.

[17] M. Casini, A. Garulli, A. Vicino, Input design for worst-case system identification with uniformly quantized measurements, in: Proc. of SYSID 2009, Saint-Malo, France, pp. 54-59.

[18] M. Casini, A. Garulli, A. Vicino, Input design in worst-case system identification using binary sensors, IEEE Trans. Automatic Control 56 (2011) 1186-1191.

[19] L. Y. Wang, G. G. Yin, J. F. Zhang, Y. Zhao, System Identification with Quantized Observations, Control: Foundations \& Applications Systems \& control, Springer, 2010.

[20] V. Cerone, Feasible parameter set for linear models with bounded errors in all variable, Automatica 29 (1993) 1551-1555.

[21] V. Cerone, D. Piga, D. Regruto, Set-membership error-in-variables identification through convex relaxation techniques, IEEE Transactions on Automatic Control 57 (2012) 517-522.

[22] V. Cerone, D. Piga, D. Regruto, Enforcing stability constraints in set-membership identification of linear dynamic systems, Automatica 47 (2011) 2488-2494.

[23] V. Cerone, D. Piga, D. Regruto, Improved parameters bounds for set-membership EIV problems, International Journal of Adaptive Control and Signal Processing 25 (2011) 208-227.

[24] H. Suzuki, T. Sugie, System identification based on quantized i/o data corrupted with noises and its performance improvement, in: 45th IEEE Conference on Decision and Control, pp. 3684-3689.

[25] M. Ikenoue, S. Kanae, Z. J. Yang, K. Wada, Identification of errors-in-variables models from quantized input-output measurements vai bias-compensate instrumental variable type method, International Journal of Innovative Computing, Information and Control 6 (2010) 183198.

[26] V. Krishnamurthy, Estimation of quantized linear errors-in-variables models, Automatica 31 (1995) 1459-1464.

[27] T. Başar, G. J. Olsder, Dynamic noncooperative game theory, Classics in applied mathematics, SIAM, 1999.

[28] T. Kailath, A. H. Sayed, B. Hassibi, Linear estimation, Prentice Hall NJ, 2000.

[29] A. Ben-Tal, L. El Ghaoui, A. Nemirovski, Robust Optimization, Princeton Series in Applied Mathematics, Princeton University Press, 2009. 